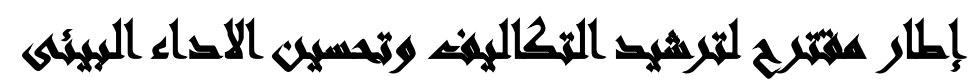

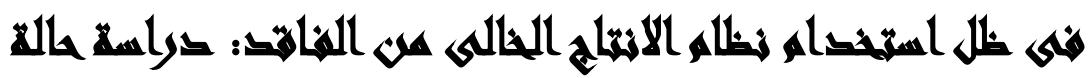 \\ [rr] \\ عمرو حسين عبد البر(')- وائل فوزى عبد الباسط(')- ريهام السبا مجدى محمد(r)

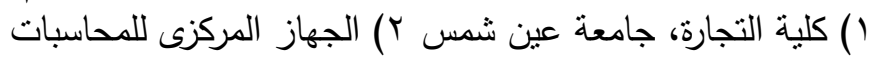 \\ المستخله
}

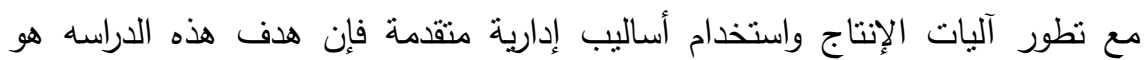

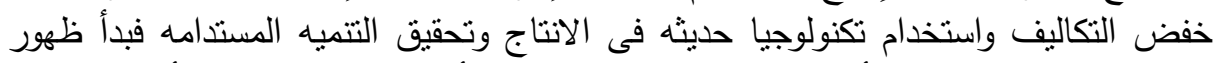

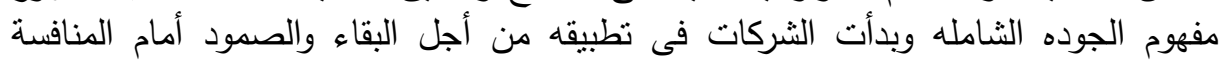

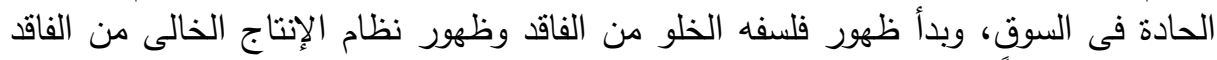

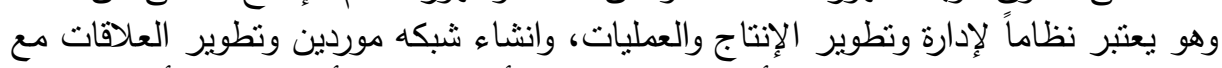

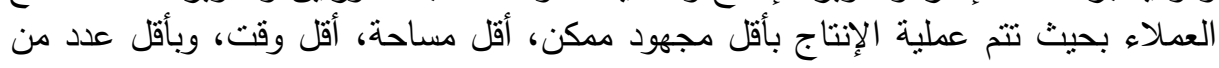

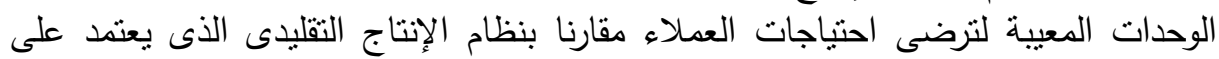
إنتاج دفعات وأحجام كبيرة.

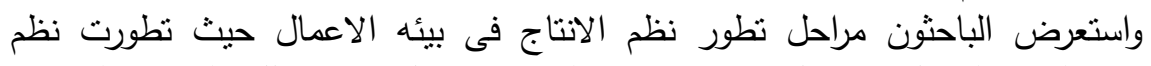

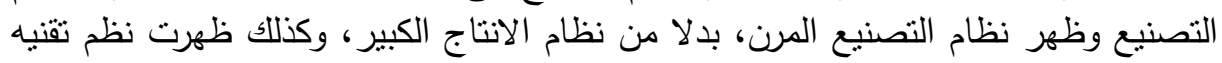

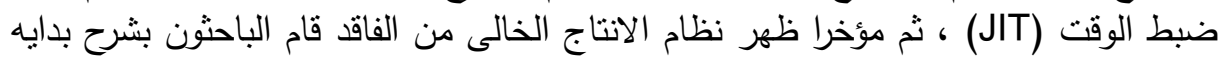

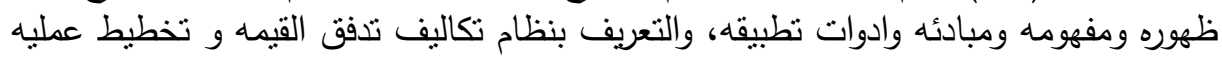

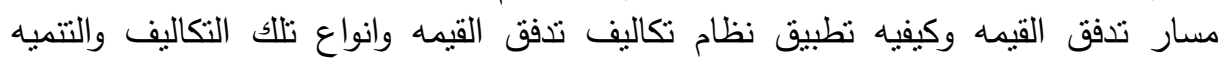

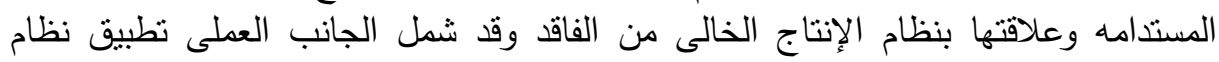

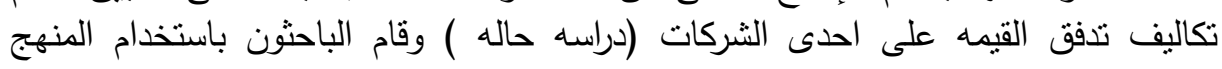

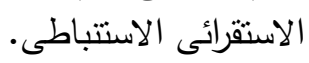

وتوصل الباحثون إلي أنه في ظل استخدام نظام الانتاج الخالى من الفاقد يعد نظام

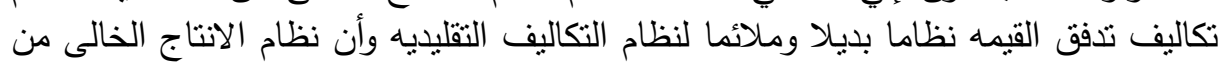

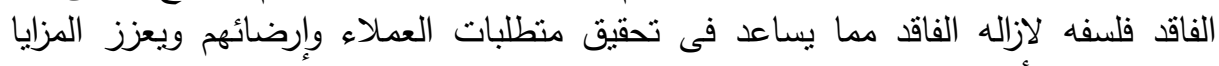

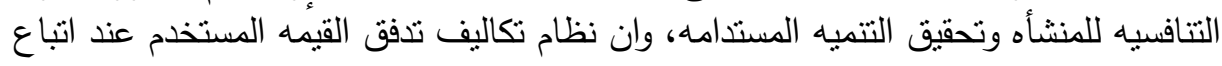

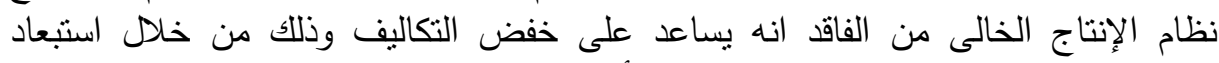

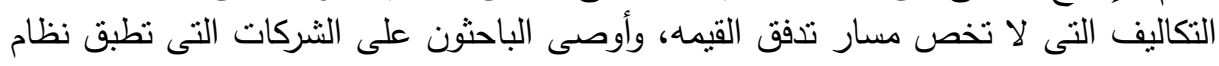

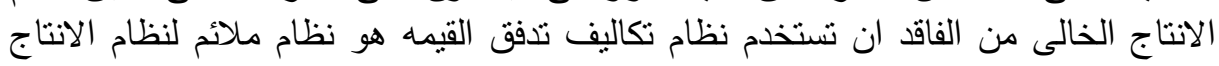
الخالى من الفاقد وذللك لسهوله ودقته

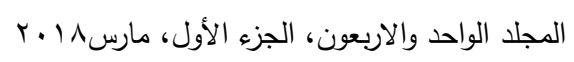




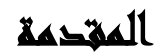

مع ظهور آليات إنتاج حديثة وأساليب إدارية متقدمة أصبح لزاما على الثركات أن تعمل على خفض التكاليف واستخدام تكنولوجيا حديثه فى الانتاج وتقليل نسب التلوث البيئى وتحسين جودة المنتج و السعى الى تحقيق التتميه المستدامه مما تطلب الأمر على الثركات العمل على تطبيق مفهوم الجودة الثشاملة على جميع الانشطه و هنا ظهرت محاسبة الخالي من الفاقد من اجل البقاء والصمود أمام المنافسة الحادة فى كل من السوق العالميه والمحليه، ويعتبر نظام الإنتاج الخالى من الفاقد والضائع ( النظام اللينى ) نظاماً خلق لتنظيم وإدارة وتطوير العمليه الإنتاجيه بحيث تتم عملية الإنتاج بأقل مجهود ممكن، بأقل رأس مال، فى أقل مساحة، وفى أقل وقت، وبأقل عدد من الوحدات المعيبة لترضى احتياجات العملاء مقارنأ بنظام الإنتاج التقليدى الذى يعتمد على إنتاج دفعات وأحجام كبيرة ويعتمد هذا النظام ايضا على انثاء شبكه موردين قويه وتطوير العلاقات مع العملاء الحالين وجذب عملاء جدد و و

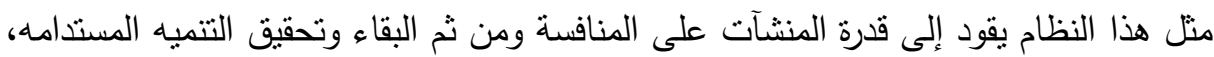

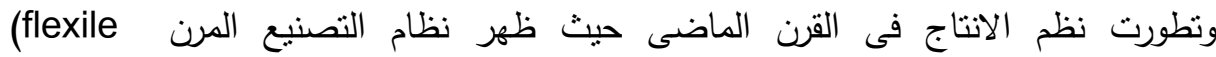

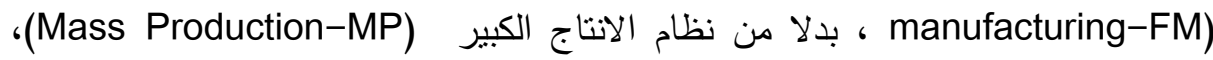
وكذلك ظهرت نظم تقنيه ضبط الوقت (JIT) ، ثم مؤخرا ظهر نظام الانتاج الخالى من الفاقد

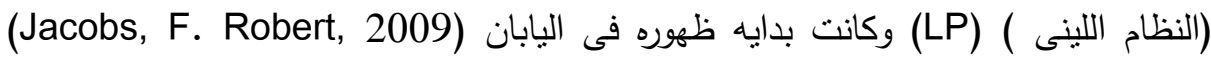
وتحديدا فى شركه تويوتا والتى كانت رائده فى تطبيق نظام الانتاج الخالى من الفاقد كتطوير

لنظام الانتاج فى الوقت المحدد (JIT) وليس بديلا له (Patricio A. Torres, 2005).

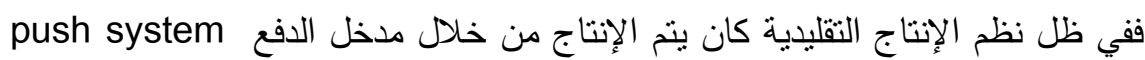

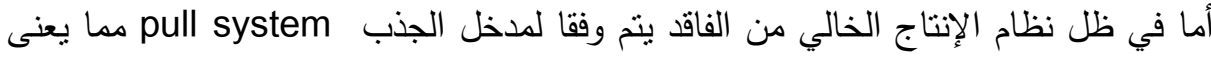
عدم الإنتاج إلا إذا وجدت مسببات قادمة من الأسواق وفقا لمواصفات محددة مسبقا مما يترتب

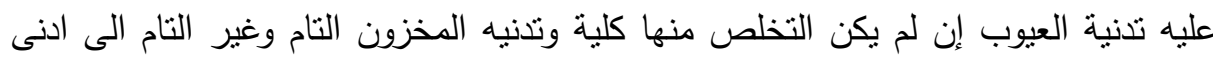
حد ممكن(محمد محسن عوض، يسرى امين ، ب ا • r). 
لقد انبثق مفهوم الخلو من الفاقد نتيجه التغيرات التى حدثت فى خطوط الانتاج حيث أصبحت بيئه التصنيع تتادى بفلسفه الخلو من الفاقد واصبحت طرق التصنيع التقليديه غير التير مواكبه للتكنولوجيا الحديثه وغير ملائمه لها، فقام مفهوم الخلو من الفاقد على ثنلاث مفاهيم

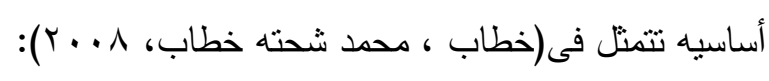

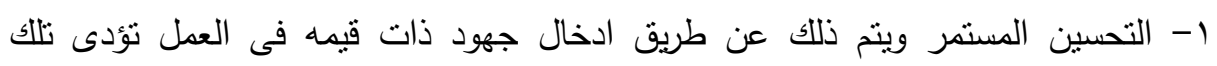
الجهود الى إحداث تغيرات حقيقيه فى التشغيل والانتاج وازال اى معوقات خلال العمليه

$$
\text { الانتاجيه }
$$

r- ازاله الفاقد يعنى ذللك التخلص من كل انواع الفاقد واشكاله أثثاء عمليات الانتاج والتشغيل r- تمكين الافراد ويتم ذلك من خلال اعطاء مستوى معين من العاملين بالمنشأه سلطه اتخاذ القرارات وينم ذلك عن طريق تدريبهم على اعلى مستوى ليكون لديهم القدره على اتخاذ

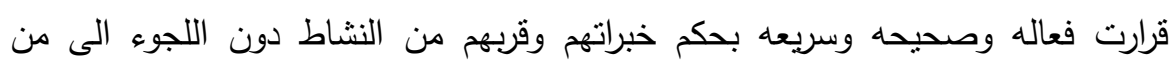
برئسهم فيمكن القول أن كل فرد يشارك فى خفض الفاقد وإجراء التحسين المستمر وتحقيق التتميه المستدامه وتحقيق رضا للعميل.

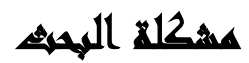

ومن خلال الدراسات السابقه التى تم عرضها بشكل تفصيلى فى الرساله ومنها دراسة

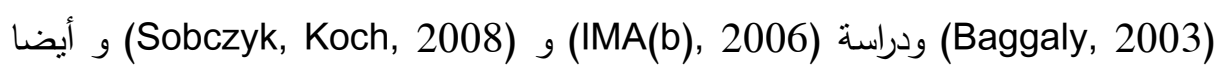
دراسه (Laura C. Maia, Anabela C. Alves, Celina P. Leão, 2012) فتم التوصل الى ان تتبع مشكلة البحث من وجود فاقد فى الوقت وفاقد في تشغيل وفاقد فى

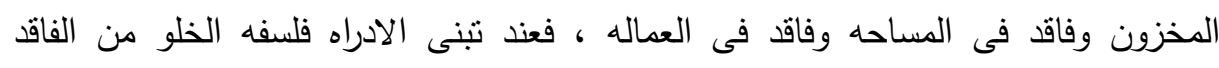

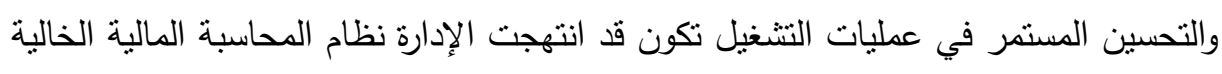

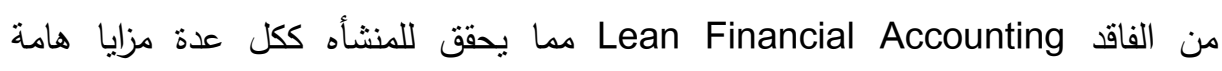
وللأطراف أصحاب المصلحة فيها وتحقيق التتميه المستدامه، تتسم بيئه الاعمال فى وقتتا هذا لـأ

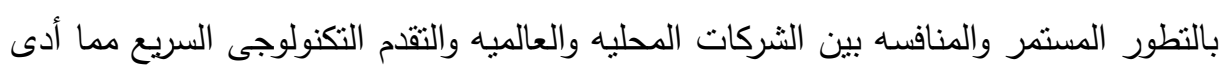

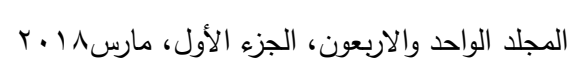


الى تطوير نظام تقنية ضبط الوقت وظهور ما يعرف بنظام الإنتاج الخالى من الفاقد Lean)

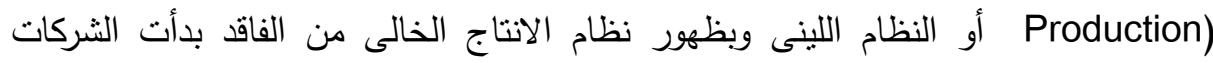
بتسليط الانظار على ماهو اهداف ومبادئ هذا النظام ولماذا نتجه الشركات الى تطبيقه.

\section{أسميلد الهيهم}

وتكمن فى السؤال الرئيسى التالى: "ما أمكانيه تخفيض التكاليف وتحقيق التتميه المستدامه

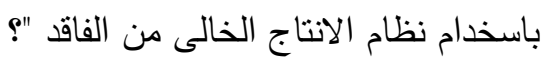
و تتفرع عده اسئله من السؤال الرئيسى وهى:

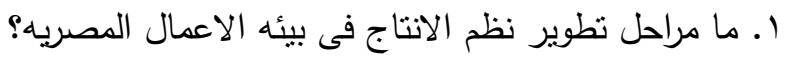

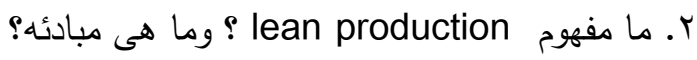
r. ما مدى تأثير تطبيق نظام الانتاج الخالى من الفاقد فى خفض استهلاك الطاقه وتحقيق التتميه المستدامه؟ ع. ما نظام تكاليف تدفق القيمه VSC ؟ و ما هى خطوات تطبيق نظام تكاليف تدفق القيمه؟ ه. ما إمكانيه تطبيق نظام تكاليف تدفق القيمه ليصبح نظام نكاليف ملائم لبيئه الاتتاج الخاليه من الفاقد؟

\section{أهمبر}

ا ـ ظهرت العديد من النظم الانتاجيه فى بيئه التصنيع التى تتادى الى تحقيق جوده المنتج من المره الأولى دون عيوب فى الصناعه خلال العقدين الاخيرين ومنها نظام الانتاج اللينى (lean production) ، والذى يرجع الفضل فى انشاءه شركه نوتوتا اليابانيه،

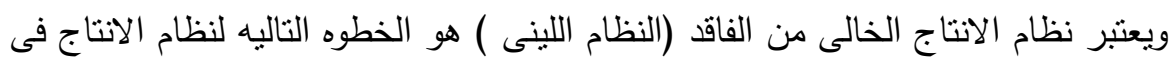
الوقت المحدد . r. ويسعى نظام الإنتاج الخالى من الفاقد الى ترشيد استهلاك الموارد داخل العمليه الانتاجيه ويتم ذلك من خلال القضاء على الفاقد بكافه اثكاله وانواعه و تحسين تدفق الموارد خلال 
مسارات تذفق القيمه ونظوير العمليه الانتاجيه والخدميه من خلال برامج التحسين المستمر وتدريب العاملين بالمنشأه والسعى الى تحقيق التتميه المستدامه وغيرها.

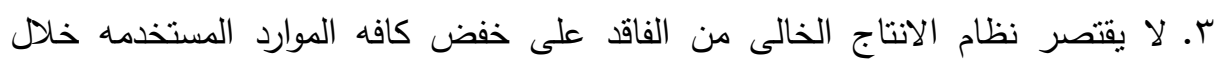

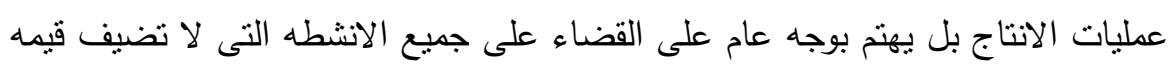
حقيقيه للعمليه الانتاجيه ولا تضيف قيمه للعميل وتقوم بازالة تلك الانشطه والتخلص منهات التها.

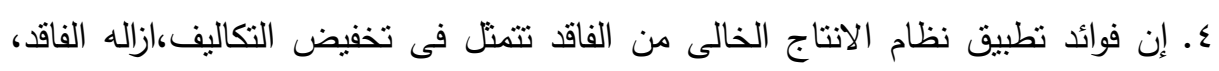

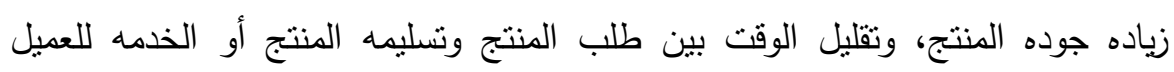

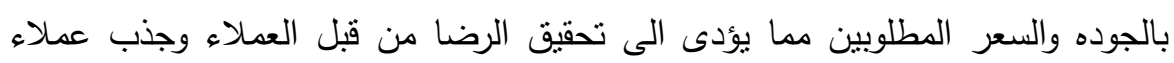
جدد، وتحقيق التتميه المستدامه.

ه. نتبع أهمية الدراسه من ندرة البحوث العربية في مجال تطبيق نظم الانتاج الخالية من الفاقد

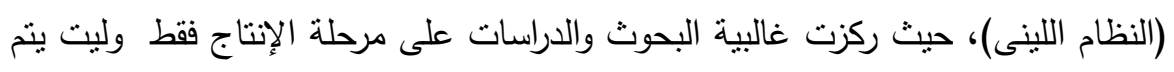

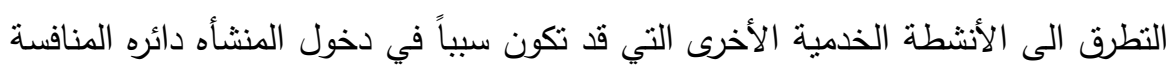

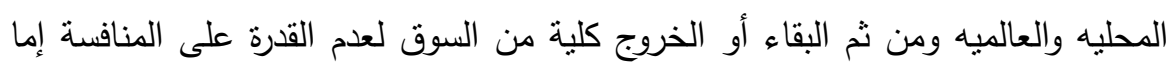

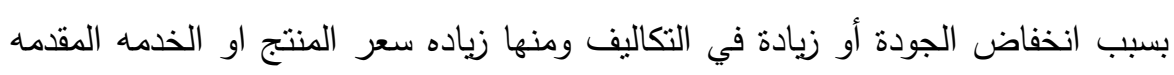
للعميل.

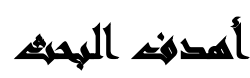

يهدف هذا البحث الى دراسه تخفيض التكاليف و التلوث الناتج عن عمليه الانتاج

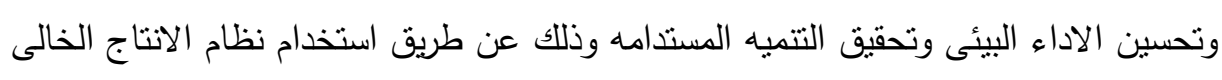
من الفاقد وهذا النظام يساعد على ترشيد استهلاك الطاقه و الفاقد بانواعه المختلفه وايضا

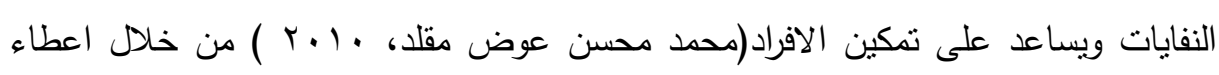

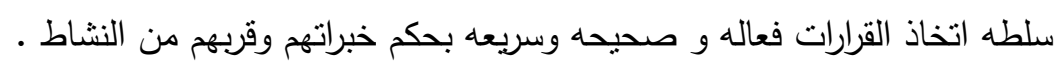
والهـف الرئيسى من ذلك الدراسه هو: " تخفيض التكاليف وتحقيق التتميه المستدامه باستخدام نظام الانتاج الخالى من الفاقد". 
ويتحقق هذا الهُف من خلال بعض الاهداف الفرعيه وهى:

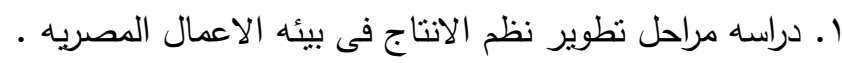

r. التعرف على مفهوم lean production وتوضيح مبادئه . r. ددى نأثير تطبيق نظام الانتاج الخالى من الفاقد فى خفض استهلاك الطاقه وتحقيق التتميه المستدامه .

ع. دراسه نظام تكاليف تدفق القيمه VSC وخطوات نطبيقه. ه. دراسه العلاقه بين تطبيق نظام تكاليف تدفق القيمه ومدى ملائمته لبيئه الانتاج الخالى من نط

\section{هزوض المهند}

يمكن صياغة فروض البحث على النحو التالى:

الفرض الأول: لا يوجد علاقه جوهريه ذات دلاله احصائيه بين تطبيق نظام الانتاج الخالى لئى

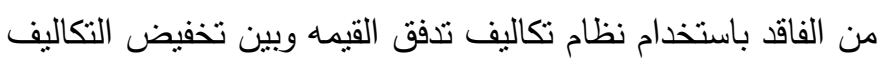

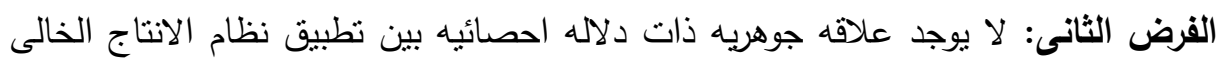
من الفاقد باستخدام نظام تكاليف تدفق القيمه وبين دقه تسعير المنتج

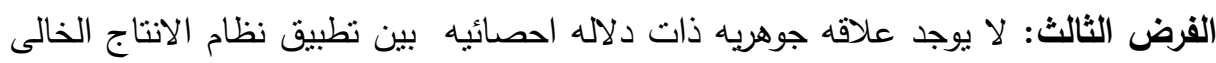

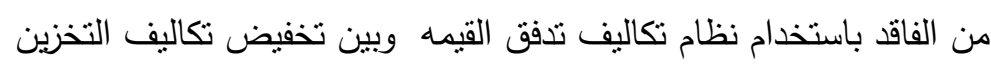
الفرض الرابع: لا نوجد علاقه جوهريه ذات دلاله احصائيه نطبيق نظام الانتاج الخالى من

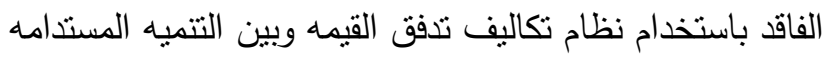




\section{التواساهت المربعية}

عرضت دراسة (Baggaly, 2003) أهم عيوب نظام التكاليف المعيارية فى ظل نظام الإنتاج الخالى من الفاقد. سعت نللك الدراسة الى التوصل الى ألى أهم عيوب نظام التكاليف المعيارية فى ظل نظام الإنتاج الخالى من الفاقد حيث أوضحت الدراسة أنه يوجد عوائق كثيره

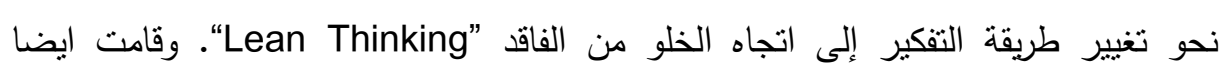
الدراسة بالتعريف بنظام تكاليف تدفق القيمة (VSC) كنظام يلائم المنشآت التى تطبق نظام الإنتاج الخالى من الفاقد (النظام اللينى ) ؛ حيث يتم حساب تكلفة مسار تدفق القيمة أسبوعياً

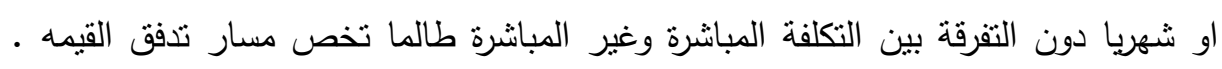
قدمت الدراسة خطوات لتطبيق نظام تكاليف تدفق القيمة، وكذلك تتاولت الدراسة كيفية التقرير عن أرباح تدفق القيمة من خلال القوائم المالية الخاصة بمسارات تدفق القيمة و كذللك كيفيه

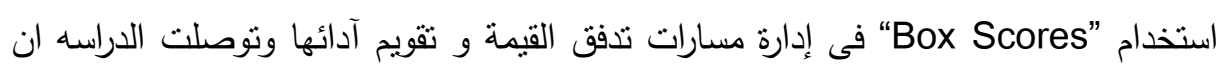
من اهم العيوب عدم دقه تكلفه المنتج • وتعرضت دراسة (IMA(b), 2006) لبعض المفاهيم النظرية المرتبطة بالمحاسبة فى ظل استخدام نظام الإتتاج الخالى من الفاقد. وتوصلت تلك الدراسة ببعض النقاط الرئيسية وتتمثل

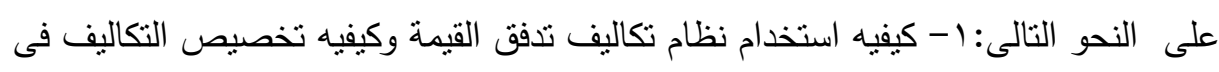
ظل نظام الانتاج الخالى من الفاقد. ب- كيفية صنع بعض القرارات مثل التسعير وقبول

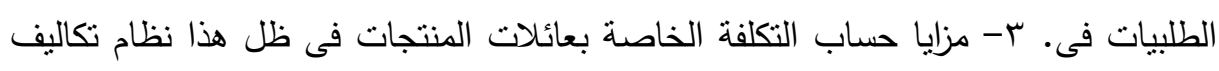
تدفق القيمه. ع - خفض العمليات التى لاتضيف قيمة للعميل . وتناولت دراسة (Sobczyk, Koch, 2008) تعرضت تلك الدراسه الى مفهوم تخطيط

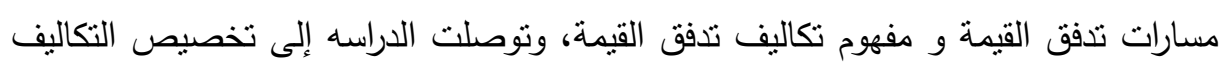
فى ظل نظام تكاليف تدفق القيمة مناسب جدا وايضا تتاولت الدراسة كيفية التعامل مع الموارد المشتركة مسارات تدفق قيمة(تكاليف مساعده). وشرحت الدراسه الى أهمية عملية تخطيط 
مجلة العلوم البيئية

معهد الدراسات والبحوث البيئية - جامعة عين شمس لهن 
(Laura C. Maia, Anabela C. Alves, Celina P. Leão, وتناولت دراسة (2012 بيئه عمل مستدامه فى ظل نظام الانتاج الخالى من الفاقد فى صناعه الغزل والنسيج والملابس الجاهزه ويهدف هذا البحث إلى تقديم نظام الانتاج الخالى من الفاقد باعتباره نموذجا

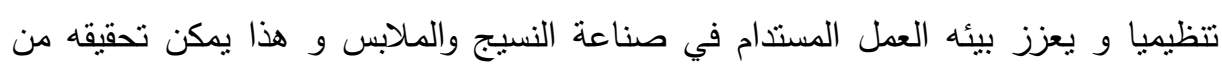
خلال بعض الأدوات والتي يمكن من خلالها تقلل من الطاقة، واستهلاك المياه، والنفايات

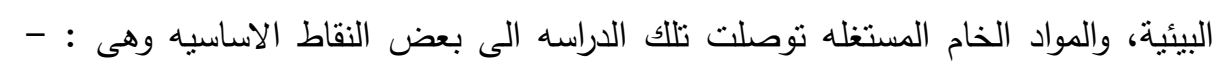

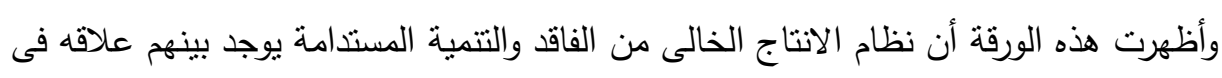

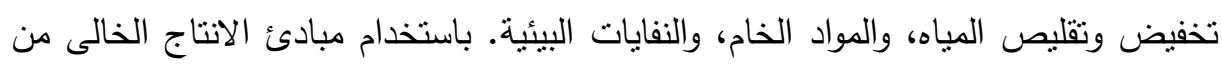
الفاقد وأدواته مثل خرئط تدفق القيمه، So،كايزين، أو غيرها، سوف تستفيد لتحقيق هذانه

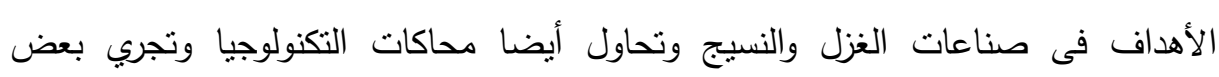
التغييرات على العمليات التقليدية . وتناولت دراسة (Fredrik Engström, 2016) مساهمه نظام الانتاج الخالى من الفاقد فى تحقيق التنميه المستدامه في صناعة الأغذية و سعت تلك الدراسة الى شرح كيف يقوم

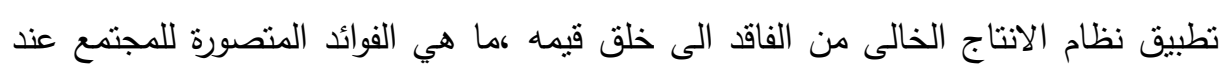

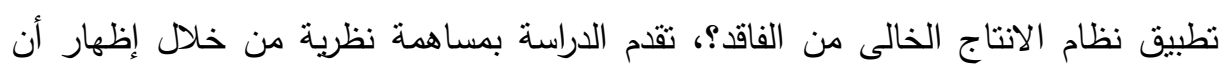

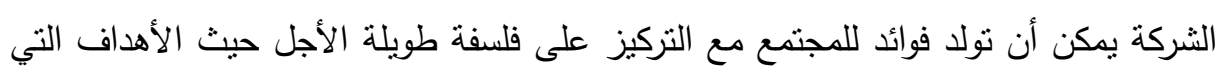

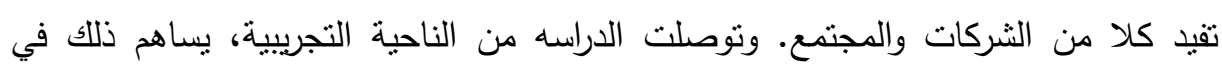
إظهار كيف يرى المديرون في صناعة الأغذية علاقه العمل بالإنتاج اللينى و تحقيق بالتتمية

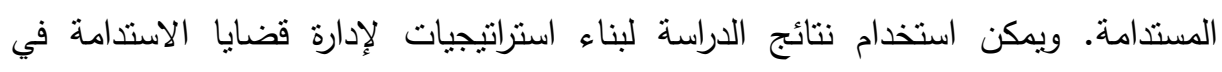
صناعة الأغذية . ومعظم الدراسات السابقه لم تطبق جميع خطوات نظام تكاليف تدفق القيمه ويعتبر هذا الدافع لاجراء هذه الدراسه الاس 


\section{مصووت اللهمث}

الحدود المكانية: بالتطبيق على شركه (ح ع ) وهى من أكبر وأهم شركات المقاولات في

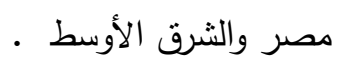

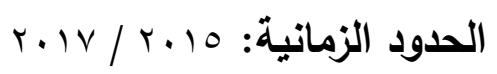

\section{منهيجي المهيد}

اعتمدت الدراسة على المنهج الإستقرائى الإستتباطى الذى يقوم على تجميع وتحليل

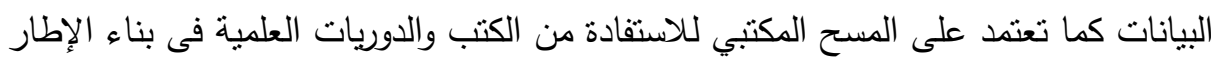

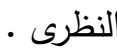

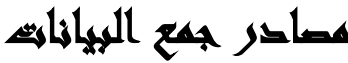

تم الإعتماد فى هذة الدراسة على مصدرين لجمع المعلومات : أ- نظام التكاليف المتبع بالقطاع محل التطبيق شركه "ح ع ع ". ب-المصادر النى إنتهجت مبدأ المسح المكتبي والدوريات والكتب والمقالات لنظام الانتاج الخالى من الفاقد، ونظام تكاليف تدفق القيمه والتتميه المستدامه .

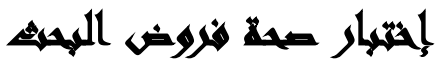

اختبار الفرض الاول: لا يوجد علاقه جوهريه ذات دلاله احصائيه بين تطبيق نظام الإنتاج الخالى من الفاقد باستخدام نظام تكاليف تدفق القيمه ويين تخفيض التكاليف . وللتحقق من صحه هذا الفرض قام الباحث بحساب التكلفه عند استخدام المنشأه النظم

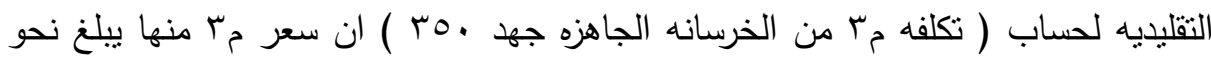
آT جنيها بينما عند استخدام نظام تكاليف تدفق القيمه لحساب (تكلفه مب من الخرسانه

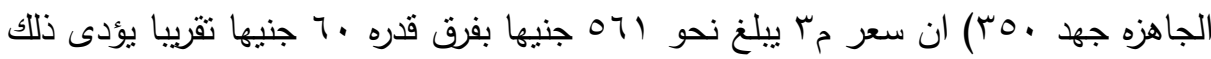

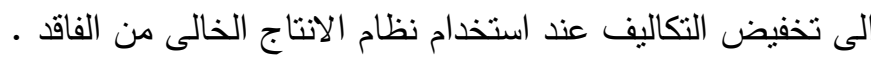

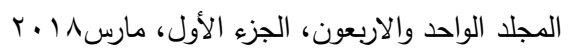


قبل قيام الباحث بعرض كيفيه قياس تكلفه مسار تدفق القيمه وفقا لنظام تكاليف تدفق القيمه سوف يقوم بعرض قياس تلك التكلفه بواسطه الطريقه المتبعه بالمنشأه محل التطبيق

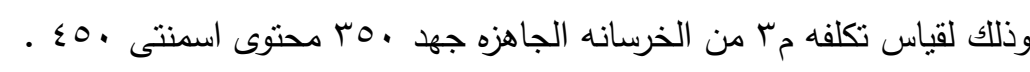

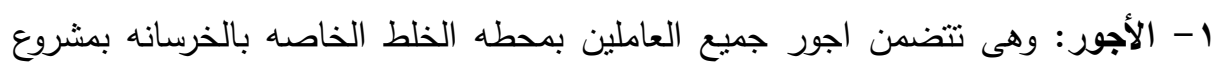

العاصمه الاداريه وكذلك العاملين بالاداره وتتحمل اجورهم على مركز تكلفه المحطه .

ץ- مستلزمات سلعيه: تتضمن تكلفه المواد الخامه المستخدمه فى انتاج الخلطه الخرسانيه

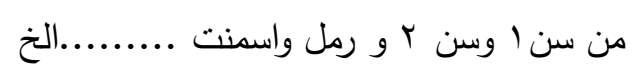

r- الورش: يشمل هذا البند كل التكاليف الناتجه عن اصلاح المعدات المستخدمه بالمحطه من مضخه وتتك المياه ومولد الكهرباء وإيجار المعدات من لودر و سيارات نقل وميكسر.

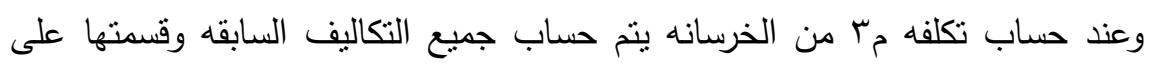

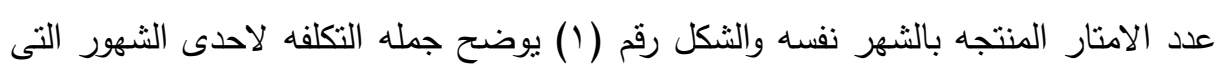

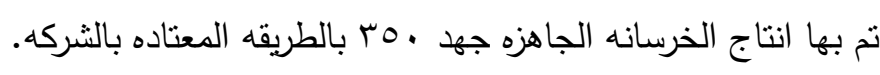

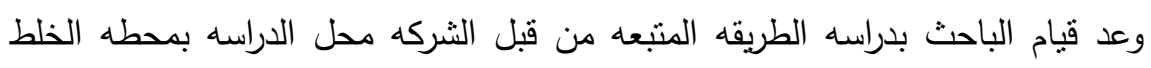

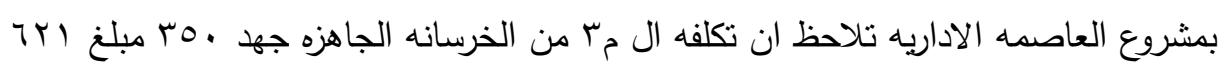

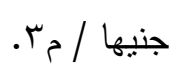

تقوم الثركه بإيجار قطعه ارض بمبلغ ... . . . جنيها شهريا وذللك لتشوين المواد الخام

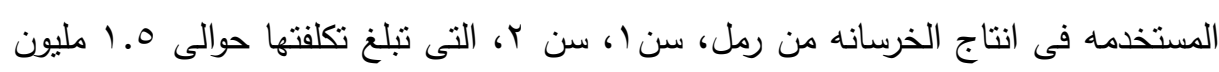
جنيه شهريا ينم تخزينه لاستخدامها لانتاج الخرسانه خلال الثهر . 


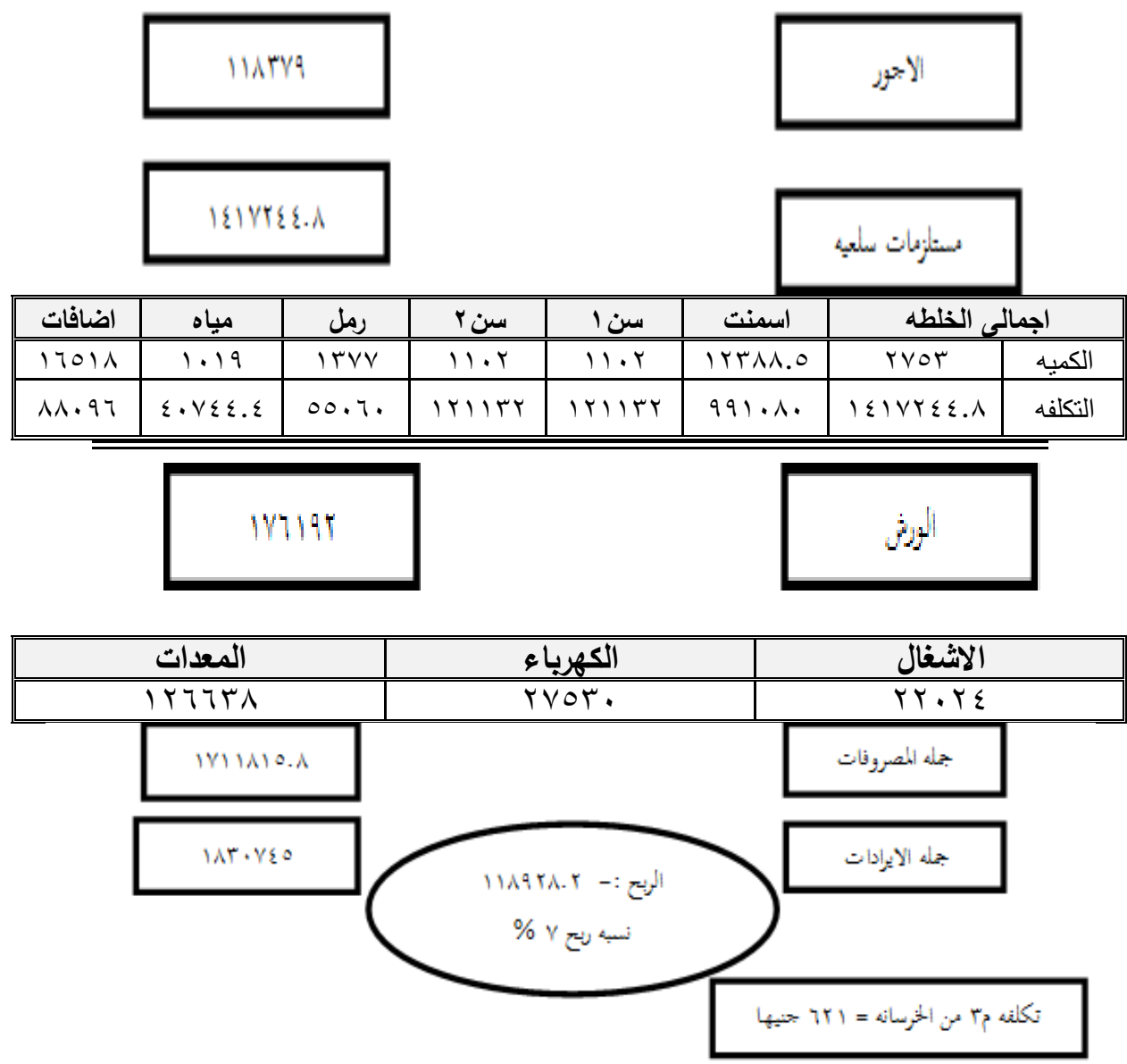

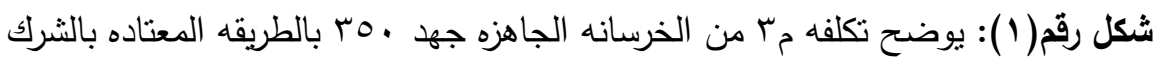

وقام الباحث بحساب تكاليف مسار تدفق القيمة عن أسبوع عمل فعلى نطبيقاً لنظام

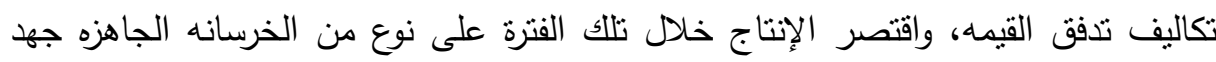
•

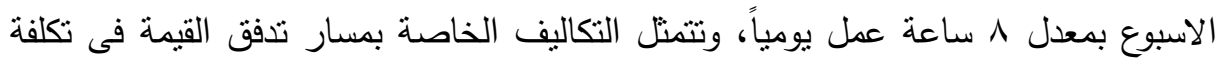


المواد، تكلفة العمالة، تكلفة الآلات، تكلفة التسهيلات،التكاليف الداعمه للانتاج والتكاليف الأخرى المرتبطة بمسار تدفق القيمة. وفيما يلى سيتم حساب تلك التكاليف تفصيلا كما هو

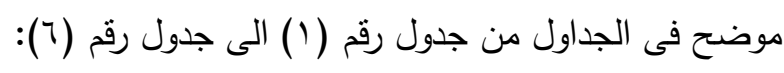

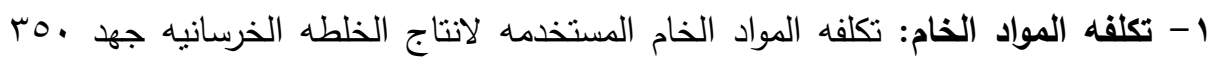
خلال اسبوع باعتبار متوسط الاتتاج اليومى كميه ـ10 م من الخرسانه ويمنل الجدول رقم

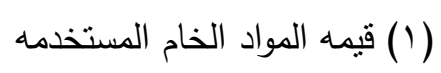

\begin{tabular}{|c|c|c|c|}
\hline التكلقه بالجنيه & سعر الكيلو من النخام & 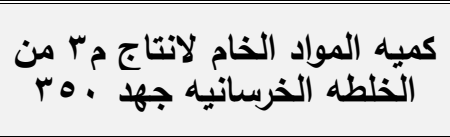 & 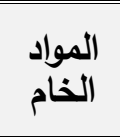 \\
\hline קצ. & A.. & •0ء. كجم & اسمنت \\
\hline$\varepsilon \varepsilon$ & 11. & ع. ـر" & سن ا \\
\hline$\leqslant \varepsilon$ & 11. & ع.. م'r & سنץ \\
\hline$r$. & $\varepsilon$. & 0. مَ" & رمل \\
\hline $1 \leq . \wedge$ & $\varepsilon$. & ע" م. & مباه \\
\hline r & $\varepsilon$ & ^ لتر & اضنافات \\
\hline $01 \leqslant .1$ & & الاجمال & \\
\hline
\end{tabular}

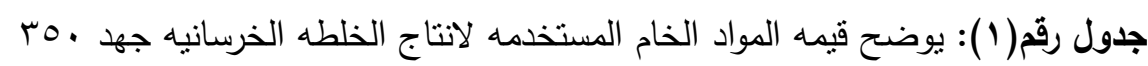

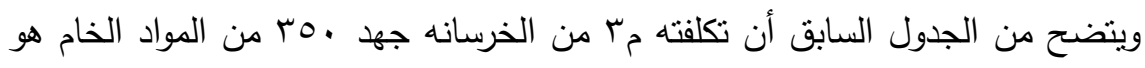

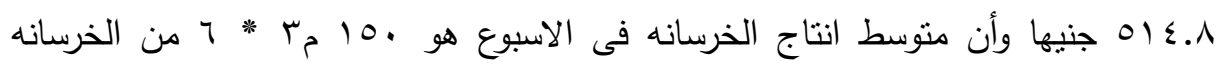

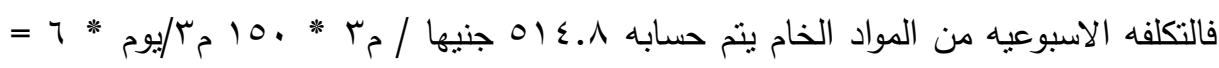
أحس

r- أجور العاملين: يتم احتساب أجور العاملين داخل مسار تدفق القيمه فقط من مهندسين

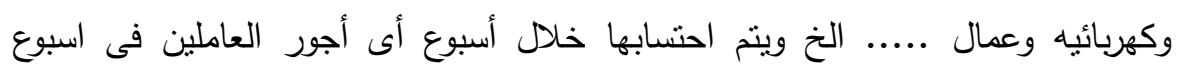

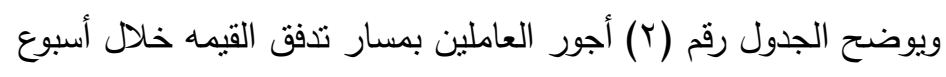


جدول رقم(ץ): يوضح أجور العاملين خلال أسبوع بمسار تدفق القيمه

\begin{tabular}{|c|c|c|c|c|}
\hline 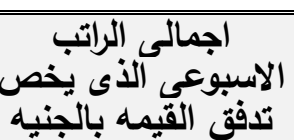 & اللراتب بالجنبوعى & للفرذّ بالجنيه الثهري & العدد & العماله داخل \\
\hline $1 \% 0$. & TYO. & $0 \ldots$ & 1 & مهندس \\
\hline Vo. & VO. & $r \ldots$ & $T$ & كهربائي \\
\hline VO. & Vo. & $r \ldots$ & $T$ & ميكانيك \\
\hline ro.. & $0 \ldots$ & $r \ldots$ & 0 & عامل \\
\hline OYO. & & & الاج & \\
\hline
\end{tabular}

فقد بلغت قيمه اجور العاملين داخل مسار تدفق القيمه خلال اسبوع مبلغ .0Yo جنيها r- تكلفه الالات والمعدات المستخدمه: يمكن حساب تكلفه استخدام الالات خلال اسبوع التى

$$
\text { تتمنل فى الجدول رقم (r) ويتم حسابها كلاتى: }
$$

أ- إهلاك الالات المستخدمه: بالاطلاع على دفاتر الثركه بلغت تكلفه لالات المستخدمه فى

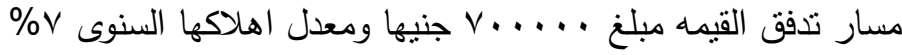

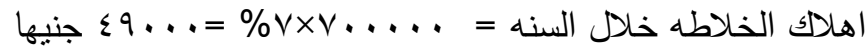

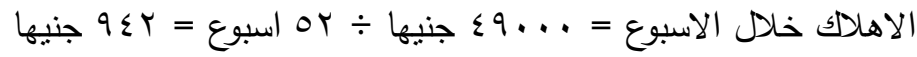
ب - صيانه الآلات المستخدمه: تقدر تكاليف الصيانه السنويه للالات المستخدمه فى الانتاج مبلغ . . . 70 جنيها سنويا اى ان التكلفه الاسبوعيه للاهلاك هى الصيانه السنويه ؛

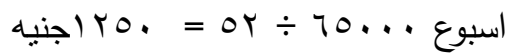

\begin{tabular}{|c|c|}
\hline التكلفه & البي البــــان \\
\hline ج $9 \leq Y$ & اهلاك الالات خلال اسبوع \\
\hline إن. & الصيانه للالات خلال اسبوع \\
\hline r 19 ا جنيها & اجمالى تكلفه استخدام الالات التى تخصل الخيه مسار تدفق \\
\hline
\end{tabular}

جدول رقم(ب): يوضح تكلفه استخدام الآلات والمعدات خلال اسبوع بمسار تدفق القيمه

ع - تكلفه التسهيلات: ويمنل الجدول (ع) تكلفه التسهيلات داخل مسار تدفق القيمه لاتتاج

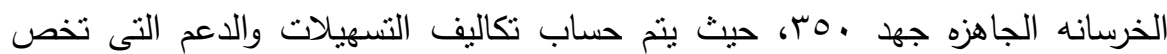
بشكل مباشر مسار تدفق القيمه وهى تتمنل فى الايجار التمويلى، ايجار المبانى، الكهرباء وتكلفه الالات المساعده فى العمليه الإنتاجيه.

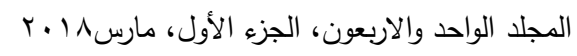


أ- تكاليف استخدام الآلات مساعده للآلات الإنتاحيه: تكلفه إيجار معدات بالموقع خلال أسبوع : تكلفه ايجار اللودار =

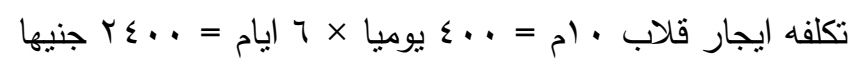

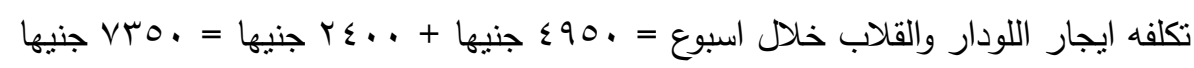

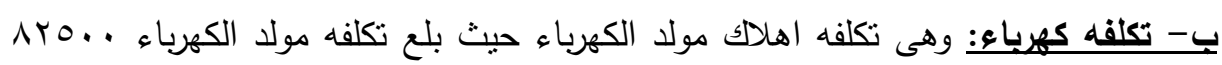
جنيها

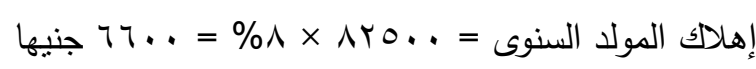

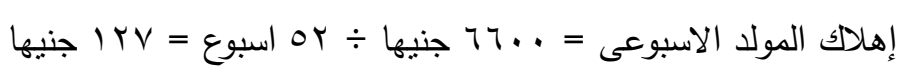
جدول رقم(ع ): يمنل تكلفه التسهيلات لمسار تدفق القيمه لانتاج الخرسانه

\begin{tabular}{|c|c|}
\hline التكلفه & البيــــان \\
\hline 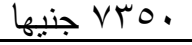 & تكاليف استخدام الالات مساعده للالات الانتاجيه \\
\hline V & تكلفه كهرباء \\
\hline جنيه V V جV & تكلفه التسهيلات \\
\hline
\end{tabular}

ه- تكاليف اخرى: والجدول رقم (0) يمثل إجمالى التكاليف الأخرى داخل مسار تدفق القيمه

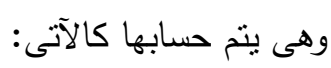

أ - تكلفه الوقود المستخدم لتشغيل مولد الكهياء بمسار تدفق القيمه: وذلك يمكن حساب تكلفه الوقود خلال اسبوع كما يلى: كمية الوقود التى تم إدداد مولد الكهرباء بها خلال الأسبوع باللتر × سعر اللتر من الوقود 10.= ب - تكاليف نقل الخلطه الخرسانبه الى العمبل: يتم احتساب تكاليف نقل مَّ من الخرسانه الجاهزه مبلغ · ب جنيها للمتز تكاليف نقل الخلطه الخرسانيه الى العميل = تكلفه نقل مَّ من الخرسانه ×عدد مَّ خلال اليوم

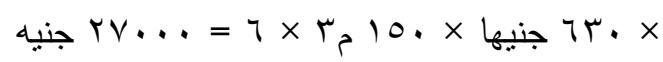


جدول رقم(•): يمثل اجمالى التكاليف الاخرى داخل مسار تدفق القيمه

\begin{tabular}{|c|c|}
\hline التكلفه & البيان \\
\hline (جنيه & تكلفه الوقود المستخدم لنتشغيل مولد الكهرباء بمسار تدفق القيمه \\
\hline T TV... & تكاليف نقل الخلطه الخرسانيه الى العميل \\
\hline جنيه TV ج ج & اجمالى التكاليف الاخرى \\
\hline
\end{tabular}

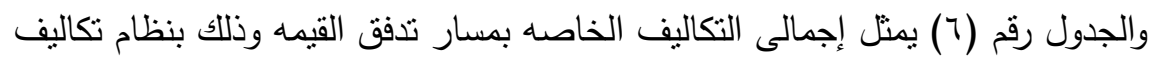

تدفق القيمه وهى تكلفه المواد، تكلفه العماله، تكلفه الالات والمعدات، تكلفه التسهيلات والدعم

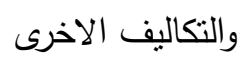

جدول رقم(7) : يمنل إجمالى تكاليف مسار تدفق القيمه وذللك بنظام تكاليف تدفق القيمه

\begin{tabular}{|c|c|}
\hline التكلفه بالجنيه & البيإ_ـــــان \\
\hline . & تكلفه المواد الخام \\
\hline جن Oro. & تكلفه العماله ( الاجور ) \\
\hline l & تكلفه الالات والمعدات \\
\hline جنيها V V جV & تكلفه التسهيلات \\
\hline l & ت تكاليف اخرى \\
\hline 促 0.07199 & جمالى تكاليف مسار تدفق القيمه خلال اسبوع \\
\hline
\end{tabular}

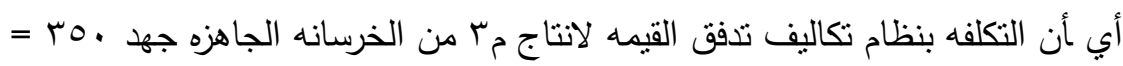

$$
\text { جنيها } 071 . \Lambda=r_{3} 9.07199
$$

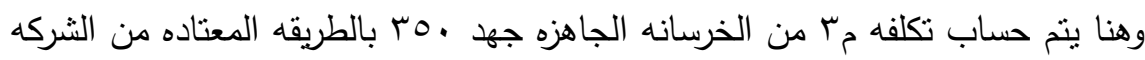

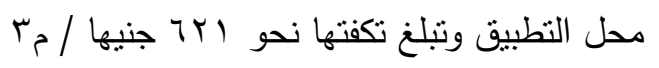

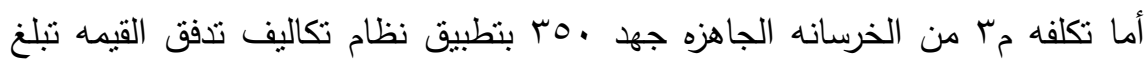
تكلفتها 1.1.07 جنيها

ويتضح أن كافه الاختبارات التى تم استخدامها فى التحقيق من الفرض تدل على رفض

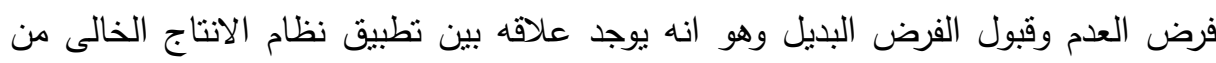
الفاقد باستخدام نظام تكاليف تدفق القيمه و و بين تخفيض التكاليف. اختبار الفرض الثانى: لا يوجد علاقه جوهريه ذات دلاله احصائيه بين نطبيق نظام الانتاج الخالى من الفاقد باستخدام نظام تكاليف تدفق القيمه وبين دقه تسعير المنتج 


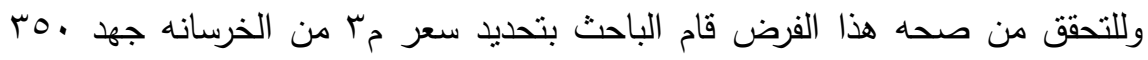

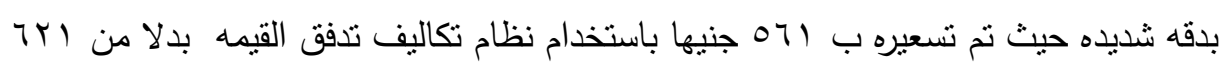
جنيها مما يؤدى الى دقه نسعير المنتج عند استخدام نظام الانتاج الخالى من الفاقد وتحديد

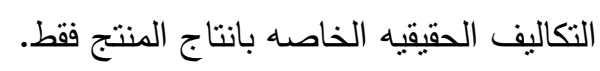

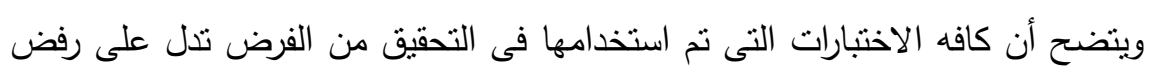
فرض العدم وقبول الفرض البديل وهو أنه يوجد علاقه بين نطبيق نظام الانتاج الخالى من الفاقد باستخدام نظام تكاليف تدفق القيمه ودقه تسعير المنتج اختبار الفرض الثالث: لا يوجد علاقه جوهريه ذات دلاله احصائيه بين نطبيق نظام الانتاج الخالى من الفاقد باستخدام نظام تكاليف تدفق القيمه وبين تخفيض تكاليف التخزين

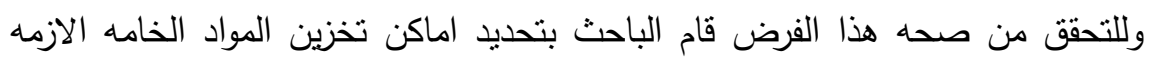

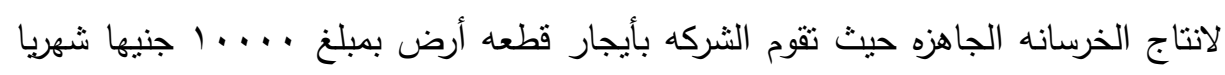

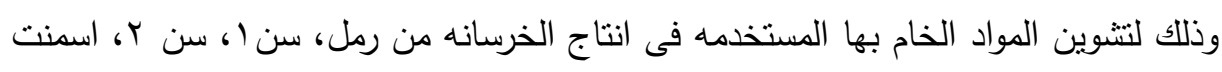

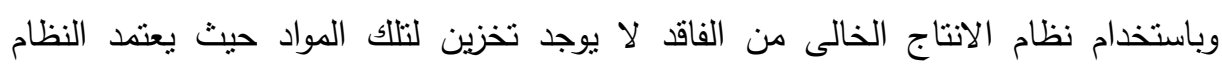

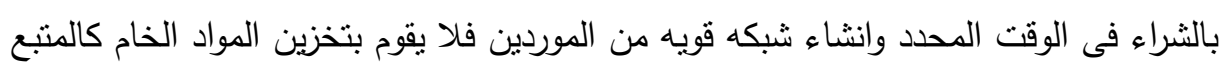
بالاساليب التقليديه مما يؤدى الى تحقيق التتميه المستدامه.

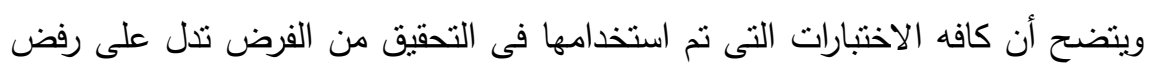
فرض العدم وقبول الفرض البديل وهو أنه هناك علاقه جوهريه ذات دلاله احصائيه بين تطبيق نظام الانتاج الخالى من الفاقد باستخدام نظام تكاليف تدفق القيمه وبين تخفيض الفي تكاليف التخزين. اختبار الفرض الرابع: لا توجد علاقه جوهريه ذات دلاله احصائيه نطبيق نظام الانتاج الخالى من الفاقد باستخدام نظام تكاليف تدفق القيمه وبين التتميه المستدامه. وللتحقق من صحه هذا الفرض قام الباحث قام الباحث بالرجوع للمعمل الخاص بالثركه محل الدراسه ( مععل اختبار الخرسانه الجاهزه ) تلاحظ انه يقوم باختبار عينه من خلطه

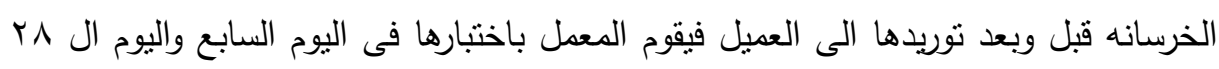
بعد الصب ويقوم بتكسير مكعبات من الخرسانه الجاهزه (

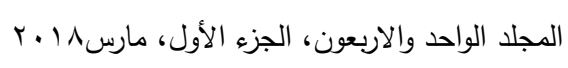


باستخدام الات ضغط معينه وتحديد مدى قوه الخرسانه ومتوسط مايقوم به المعمل اسبوعيا من اختبار للخرسانه كما افاد مهندس المعمل هو .11 مكعب اسبوعيا ومتوسط تكلفه المكعب الواحد = 7 جنيها. التكلفه الاسبوعيه للمكعب = عدد المكعبات الاسبوعيه × تكلفه المكعب الواحد

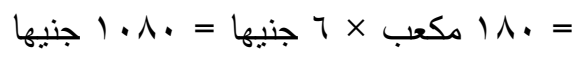

مع تطبيق نظام الانتاج الخالى من الفاقد نقوم بأعاده تدوير نتائج اختبارات المعامل

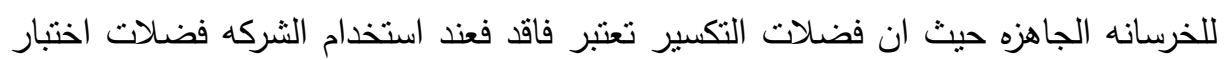
الهدم عن طريق تفتيت مكعبات الخرسانه بعد الاختبار و استخدامها فى صناعه الاسفلت

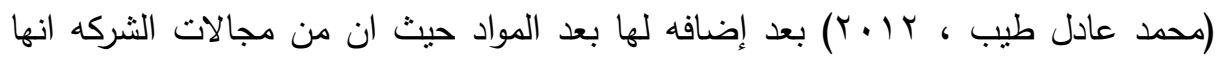
تقوم بعمل خلطات اسفلتيه مما يساعدها على استخدامها واعاده تدويرها مره اخرى والاستفاده منها حفاضا على تحقيق التتميه المستدامه. ويتضح أن كافه الاختبارات التى تم استخدامها فى التحقيق من الفرض تدل على رفض هض فرض العدم وقبول الفرض البديل وهو انه هناك علاقه جوهريه ذات دلاله احصائيه بين تطبيق نظام الانتاج الخالى من الفاقد باستخدام نظام تكاليف تدفق القيمه وبين التتميه

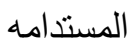

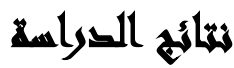

توصلت الدراسة إلى النتائج التالية :

• توصلت الدراسه انه يوجد علاقه جوهريه ذات دلاله احصائيه بين تطبيق نظام الانتاج الخالى من الفاقد باستخدام نظام تكاليف تدفق القيمه وبين تخفيض التكاليف. • توصلت الدراسه أنه توجد علاقه جوهريه ذات دلاله احصائيه بين استخدام نظام التكاليف تدفق القيمه ودقه تسعير المنتج • • نوصلت الدراسه أنه يوجد علاقه جوهريه ذات دلاله احصائيه بين تطبيق نظام الانتاج الخالى من الفاقد باستخدام نظام تكاليف تدفق القيمه وبين تخفيض تكاليف التخزين. 


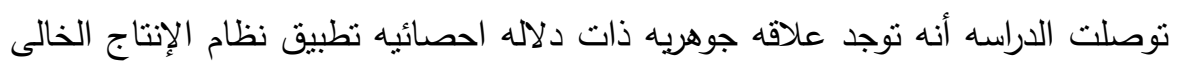
من الفاقد باستخدام نظام تكاليف تدفق القيمه وبين التتميه المستدامه.

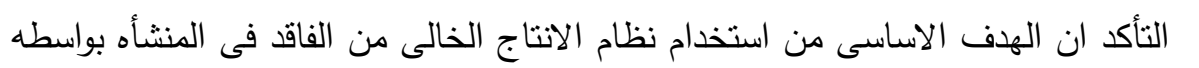

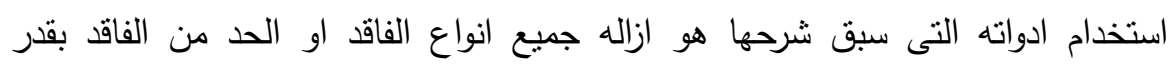
المستطاع.

يقوم النظام اللينى على مبادئ اساسيه خمسه وهم: نظام السحب، تمكين العاملين، تعظيم

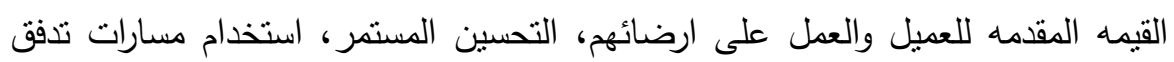

• يعتبر نظام الانتاج الخالى من الفاقد فلسفه لازاله الفاقد مما يساعد فى تحقيق متطلبات العملاء وارضائهم مما يعزز المزايا التتافسيه للمنشأه.

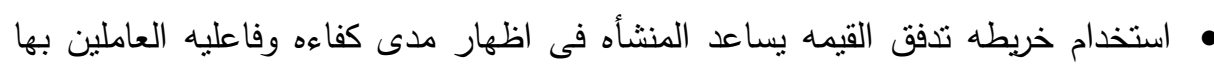
ومدى مشاركتهم ومرونتهج و قدرتهم ومدى دعم الاداره العليا. • فى ظل استخدام المنشاه نظام الاثتاج الخالى من الفاقد يعد نظام تكاليف تدفق ونق القدام القيمه

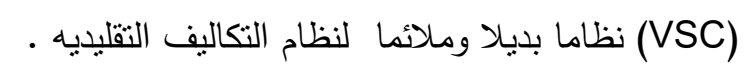
• عندما تدار المنشأه من خلال تدفق القيمه فذلك يؤدى الى تحول نظام التكاليف فالمنشأه

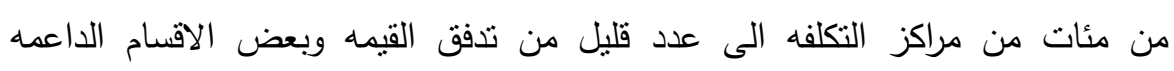

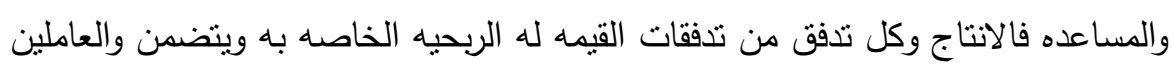
به والمسؤلين عنه بدايتا من استلام طلب العميل الى شحن البضاعه الى العميل واستلام الفاتوره.

• توافر بعض المنطلبات بالمنشأه من اجل تحقيق فكر التتميه المستدامه بابعادها المختلفه

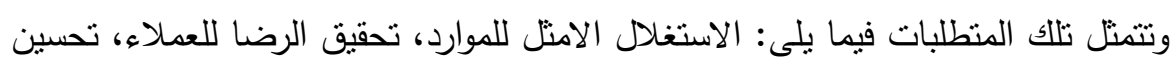

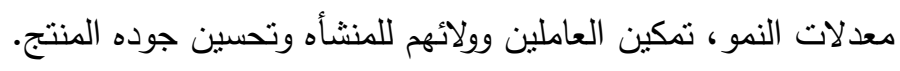




\section{توكيامش التراسم}

$$
\text { • • يوصى الباحث بما يلى: }
$$

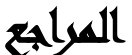

دراسه حول حمايه البيئه من خلال اعاده تدوير الركام الخرسانى فى الخلطات الاسفلتيه،

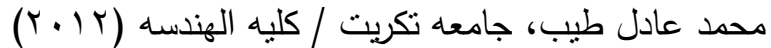

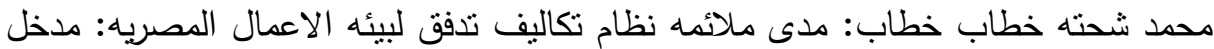

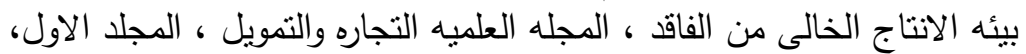

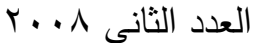

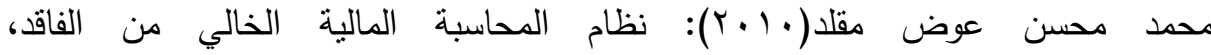
www.acc4arab.com

Jacobs, F. Robert; et.al.( 2009) :“Operations \& Supply Management”, McGraw-Hill Companies Inc., New York. 6th Edition,

Improving production performance through lean manufacturing techniques and performance in lean concepts ,Patricio A. Torres, M.B.A. Matthew P. Stephens, PHD(2005)

El-Adawy, Laila Mohamed El-Shawadfy(2010): "Improving Performance Measurement Framework to be Convent to Lean Environment", Unpublished Thesis, Tanta University

Zimmerman, Jerold L., (2009) "Accounting for Decision Making \& Control”, McGraw-Hill, New York, 6th Edition

Peter Hines, Matthias Holweg and Nick Rich t , (.2017) Learning to evolve A review of contemporary lean thinking www.emeraldinsight.com/researchregister Vol. 24 No. 10

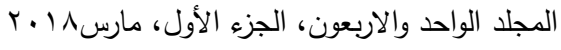


Kocakulah; Mehmet C., A. David Austill, Daniel E. Schenk, (March/April 2011), "Lean Production Practices for Efficiency”, Cost Management, ( Vol 25, No.2).

Denish B.Modi1, Hemant Thakkar, Lean Thinking(March 2014): Reduction of Waste, Lead Time, Cost through Lean Manufacturing Tools and Technique www.ijetae.com (ISSN 2250-2459, ISO 9001:2008 Certified Journal, Volume 4, Issue 3,

\title{
A SUGGESTED FRAMEWORK FOR REDUCING COST AND LEVERAGING BETTER ENVIRONMENTAL PERFORMANCE THROUGH LEAN PRODUCTION: CASE STUDY
}

Abdel Bar, A. H. ${ }^{(1)}$; Abdelbaset,W. F. ${ }^{(1)}$ and Eltahan, Reham, E. ${ }^{(1)}$ 1) Faculty of Commerce, Ain Shams University 2) Central Auditing Organization

\begin{abstract}
With the development of production mechanisms and the use of advanced management methods, the first goal of the study is to reduce costs and use of modern technology in production and achieve sustainable development; and this was the motive of the researcher to do this reseach. Companies began to implement the total quality concept in order to survive and achieve competitive advantage in the global market. It is a system for managing and developing production and operations, establishing a network of suppliers and developing relations with customers so that the production process is done with minimal effort, less space, less time and with the least number of defective units to satisfy their customers' needs as compared to mass production system.
\end{abstract}

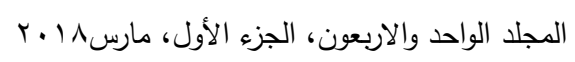




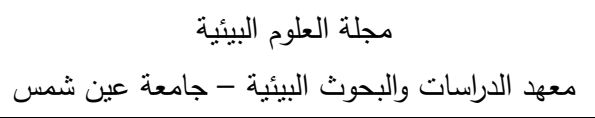

The researchers studied the stages of development of the production systems in the business environment, where as the manufacturing systems developed and appear the flexible manufacturing system instead of the mass production system then appear JIT system. The researchers then explained the beginnings of the lean production system, its concept, principles and tools. The researchers introduced the value stream cost (VSC), the process of the value stream, and how to apply the value steam cost and types of those costs are also explained by sustainable development. The researchers applied the value steam cost to a company (a case study)

Finally, the study concluded that while using the lean production, the VSC system is an alternative and suitable for the traditional cost system. The aime of lean production is to remove the waste which helps in satisfy their customers' needs, thus enhancing the competitive advantages of the establishment and achieve sustainable development. The value steam cost which use when following lean production, it helps to reduce costs by eliminating the costs that do not pertain to the value stream.

The researcher recommended that companies using lean production should use a value stream cost that is an appropriate system for lean production for ease and accuracy. 